\title{
Modelagem da infiltração em solos com encrostamento superficial. Parte I: Modelo GAML para solos estratificados
}

\author{
João H. Zonta ${ }^{1}$, Mauro A. Martinez ${ }^{2}$, Fernando F. Pruski ${ }^{2}$, \\ Demetrius D. da Silva ${ }^{2} \&$ Marcelo R. dos Santos ${ }^{3}$
}

\begin{abstract}
RESU MO
N este trabalho a meta foi avaliar o modelo de G reen-Ampt-M ein-Larson (GAM L) e GAM L modificado por Moore (1981) na simulação da infiltração de água em solos com encrostamento superficial. O s ensaios de infiltração foram realizados em Cambissolo sem cobertura, com quatro perfis de precipitação aos quais foram aplicadas três precipitações sucessivas para cada perfil, espaçadas $24 \mathrm{~h}$. Para a inclusão da camada de encrostamento superficial no modelo de GAML, o valor da condutividade hidráulica na zona de transmissão $(\mathrm{Kt})$ foi igual à taxa de infiltração estável (Tie) obtida em solo nu; no modelo de GAM L modificado por Moore (1981), foram propostas uma camada de crosta com espessura de $5 \mathrm{~mm}$ e condutividade hidráulica da crosta saturada $(\mathrm{KC})$ determinada a partir dos dados experimentais e igual a 10 e $25 \%$ da condutividade hidráulica do solo saturado $\left(\mathrm{K}_{0}\right)$. N as segunda e terceira precipitações as combinações que não consideraram o encrostamento superficial não apresentaram bons resultados, superestimando a taxa de infiltração ao longo do tempo. Para o modelo de GAML os mel hores resultados foram obtidos com uso de $\mathrm{Kt}=$ Tie determinada em solo nu, enquanto no modelo GAML se obtive, para solos estratificados,valor de $\mathrm{Kc}=10$ e $25 \%$ de $\mathrm{K}_{0}$.
\end{abstract}

Palavras-chave: condutividade hidráulica da crosta saturada, espessura da crosta, taxa de infiltração estável

\section{Modelling of the soil water infiltration in crusting soil. Part I: GAML model for layered soils}

\begin{abstract}
The aim of this study was to evaluate the Green-Ampt-M ein-Larson (GAML) model and GAML model modified by Moore (1981) for layered soils, in the simulation of water infiltration process in crusting soil. The infiltration tests were performed on a Cambisol without coverage and three successive precipitations were applied at intervals of $24 \mathrm{~h}$. For the inclusion of the soil crust layer in the input parameters of the GAM L model, the value of hydraulic conductivity in the transmission zone (Kt) was equal to stable infiltration rate (Tie) obtained in bare soil, and in the GAML model for layered soils, a surface layer (crust) were proposed with a thickness of $5 \mathrm{~mm}$ and soil crust saturated hydraulic conductivity $(\mathrm{Kc})$ determined from experimental data, and equal to 10 and $25 \%$ of the saturated hydraulic conductivity $\left(K_{0}\right)$. In the second and third rainfall, the combinations without consideration of crust did not achieve good results and overestimated infiltration rate over time. For the model of GAML, the best results were obtained using Kt = Tie determined in bare soil, while in Model GAM L for layered soils, were obtained with a value of $K_{C}=10$ and $25 \%$ of $K_{0}$.
\end{abstract}

Key words: soil crust saturated hydraulic conductivity, crust thickness, stable infiltration rate

\footnotetext{
${ }^{1}$ Embrapa Algodão, Rua 0 svaldo Cruz, 1143, Centenário, CEP 58428-095, Campina G rande, PB. Fone: (83) 3182-4355. E-mail: zonta@cnpa.embrapa.br 2 Departamento de Engenharia Agrícola/U FV, Campus U niversitário, s/n, Centro, CEP 36570-000, Viçosa, M G. Fone: (31) 3899-1910. E-mail: mmauro@ufv.br; ffpruski@ufv.br; david@ufv.br

3 Instituto Federal de Educação, Ciência e Tecnologia Baiano, IFBAIANO, Distrito de Ceraíma, CEP 46430-000, Guanambi, BA. Fone: (77) 3493-2100. E-mail: marrochas@yahoo.com.br
} 


\section{INTRODUÇÃO}

A formação do encrostamento superficial é um processo importante em solos de muitas regiões tropicais e subtropicais (Souza et al., 2007), resultante de processos complexos e dinâmicos nos quais as partículas do solo são rearranjadas e consolidadas em uma estrutura superficial coesa, cuja espessura pode variar de $0,1 \mathrm{~mm}$ até $50 \mathrm{~mm}$ (Valentin \& Bresson, 1992), caracterizada por sua alta densidade, poros de diâmetros reduzidos e condutividade hidráulica do solo saturado significativamente menor do que a da camada de solo subjacente, podendo esta redução ser superior a cem vezes, influenciando o processo de infiltração (Chowdary et al., 2006).

Schaefer et al. (2002) e Brandão et al. (2006) só não detectaram a formação de encrostamento superficial em solos com 100\% de cobertura. Desta forma, a não consideração da camada de encrostamento nos modelos de infiltração pode conduzir sua superestimativa quando se trabalha em condições propícias à formação da crosta.

Dentre os modelos de simulação da infiltração, o desenvolvido por Green \& Ampt (1911) e modificado por Mein \& Larson (1973), denominado modelo de Green-Ampt-MeinLarson (GAML), devido à sua simplicidade e embasamento físico, passou a ser amplamente utilizado na estimativa da infiltração.

Para as condições de solo com cobertura vegetal, diversos trabalhos já demonstraram o bom desempenho dos modelos de GA e GAML com uso de propostas para a adequação dos parâmetros de entrada; ressalta-se que Cecílio et al. (2007) e Zonta et al. (2010) encontraram bons resultados com a combinação do uso da taxa de infiltração estável (Tie) em substituição à condutividade hidráulica do solo saturado $\left(\mathrm{K}_{0}\right)$ como valor da condutividade hidráulica na zona de transmissão (Kt) e o valor de potencial mátrico na frente de umedecimento $\left(\psi_{\mathrm{f}}\right)$ calculado com uso da equação proposta por Cecílio et al. (2007) condições em que outros autores, como Nahar et al. (2004) e Meng et al. (2006), também encontraram bons resultados com uso do modelo de GAML.

Um problema ainda atual é a determinação dos parâmetros de entrada do modelo de GAML para solos com a presença da camada de encrostamento haja vista que, neste caso, diversos autores não encontraram bons resultados, como Silva \& Kato (1997) Shukla et al. (2003) e Zonta et al. (2010).

Moore (1981) desenvolveu, a partir do modelo de GAML, outro modelo capaz de simular o processo de infiltração em perfil de solo composto de duas camadas, permitindo a simulação em solos estratificados e a inferência da camada de encrostamento superficial no modelo de GAML. Entretanto, é grande a dificuldade na determinação das características hidráulicas da camada de encrostamento superficial. Metodologias foram propostas visando à determinação da condutividade hidráulica e espessura desta camada, porém muitas delas são de difícil utilização em nível de campo, em referência à necessidade do uso de equipamentos eletrônicos e de tecnologias de alto custo.

Uma das grandes dificuldades está relacionada à dinâmica do processo de encrostamento que ainda parece não bem definida, visto a grande quantidade de fatores envolvidos.
Diversos autores, como Valentin \& Bresson (1992), Brandão et al. (2006) e Robinson \& Woodun (2008), determinaram as características hidráulicas da crosta, sendo sua espessura determinada através de análises micromorfológicas.

O presente trabalho teve como objetivo avaliar o desempenho do modelo de Green-Ampt-Mein-Larson (GAML) e GAML, modificado por Moore (1981) na simulação do processo de infiltração de água no solo, testando metodologias para determinação dos parâmetros de entrada para solos encrostados.

\section{MATERIAL E MÉTODOS}

Os ensaios de infiltração foram realizados na área experimental do Departamento de Engenharia Agrícola da UFV, cujo solo foi classificado como Cambissolo Háplico Tb Distrófico, com declividade média de $8,0 \%$. Para a realização dos ensaios utilizou-se um simulador de chuvas do tipo pendular automatizado permitindo, assim, a aplicação de precipitações com intensidade constante e variada ao longo do tempo.

Os ensaios de infiltração consistiram na aplicação, em solo nu, de chuva simulada com quatro diferentes perfis de precipitação: constante, exponencial decrescente, duplo exponencial adiantado e atrasado (Figura 1).

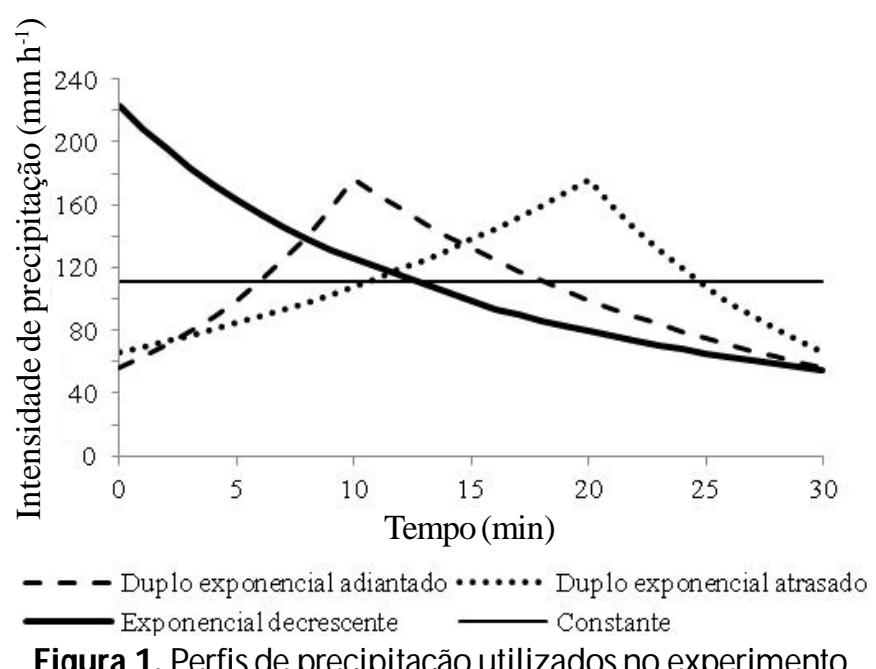

Figura 1. Perfis de precipitação utilizados no experimento

Ajustaram-se os perfis de precipitação de modo que fosse aplicada uma lâmina total de $55 \mathrm{~mm}$, tendo os ensaios duração de $30 \mathrm{~min}$, com intensidade máxima média de precipitação determinada com uso da equação de intensidade-duração e frequência para a localidade de Viçosa, MG. Foram aplicadas para cada perfil de precipitação três chuvas sucessivas com intervalos de $24 \mathrm{~h}$, sendo neste intervalo o solo mantido coberto a fim de evitar a evaporação. Foram feitas três repetições para cada perfil de precipitação.

Foram coletados os seguintes dados: conteúdo de água no solo, com uso de sondas de TDR, no início, durante e no fim dos ensaios, nas profundidades de 10, 20, 30, 40 e $50 \mathrm{~cm}$; intensidade de precipitação (ip) e taxa de escoamento superficial e, por diferença, a taxa de infiltração (Ti) de água no solo, além 
das características físicas do solo (Tabela 1), usadas para determinação de alguns parâmetros de entrada do modelo.

Tabela 1. Massa específica do solo ( $\rho)$, massa específica das partículas $\left(\rho_{\mathrm{r}}\right)$ porosidade total $(\varepsilon)$ e condutividade hidráulica do solo saturado $\left(\mathrm{K}_{0}\right)$ do perfil do solo

\begin{tabular}{|c|c|c|c|c|c|}
\hline \multirow{2}{*}{ Horizonte } & $\rho^{1}$ & $\rho_{\mathrm{r}}^{2}$ & \multirow{2}{*}{$\begin{array}{c}\varepsilon^{3} \\
\mathrm{dm}^{3} \mathrm{dm}^{-3}\end{array}$} & \multirow{2}{*}{$\begin{array}{c}\mathrm{K}_{0}{ }^{4} \\
\mathrm{~mm} \mathrm{~h}^{-1}\end{array}$} & \multirow{2}{*}{ Classe textural } \\
\hline & \multicolumn{2}{|c|}{$\mathrm{kg} \mathrm{m}^{-3}$} & & & \\
\hline A & 1,40 & 2,57 & 0,46 & 40,0 & Argilo-arenosa \\
\hline B & 1,37 & 2,58 & 0,47 & 20,0 & Argila \\
\hline
\end{tabular}

1 - Método do anel volumétrico; 2 - Método do balão volumétrico; 3 - Porosidade total; e 4 - Método do permeâmetro de carga constante

Os valores médios do conteúdo de água no solo no início dos ensaios de infiltração foram de 0,$26 ; 0,32$ e $0,33 \mathrm{~cm}^{3} \mathrm{~cm}^{-3}$ para as primeira, segunda e terceira aplicações, respectivamente.

$\mathrm{O}$ escoamento superficial produzido durante o ensaio foi conduzido para um recipiente de coleta equipado com um linígrafo, denominado Thalimedes, que registrava a altura da lâmina de água ao longo do tempo dentro do reservatório, para a determinação do volume e da taxa de escoamento superficial. Na Figura 2 é apresentado um esquema com a estrutura para realização dos ensaios, com quatro parcelas instaladas sob o simulador de chuvas e os recipientes de coleta do escoamento superficial.

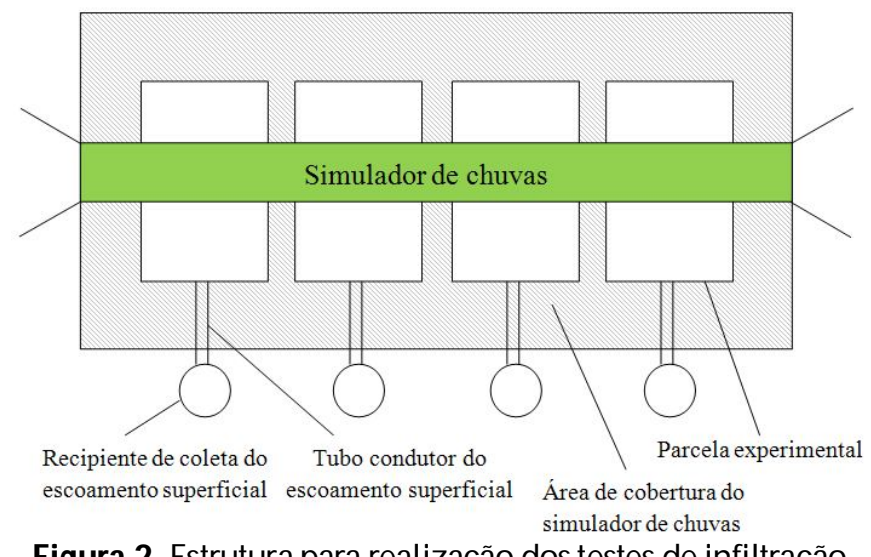

Figura 2. Estrutura para real ização dos testes de infiltração

Para a simulação do processo de infiltração de água no solo foram utilizados os modelos de GAML e GAML modificado por Moore (1981) para solos estratificados.

Considerando uma intensidade de precipitação constante maior do que a condutividade hidráulica do solo saturado, enquanto a capacidade de infiltração for maior que a intensidade de precipitação, ou seja, antes da saturação da camada superficial do solo, a taxa de infiltração é dada por:

$$
\mathrm{Ti}=\mathrm{ip}
$$

em que:

$\mathrm{Ti}$ - taxa de infiltração de água no solo, $\mathrm{mm} \mathrm{h}^{-1}$

ip - intensidade de precipitação, $\mathrm{mm} \mathrm{h}^{-1}$

A lâmina infiltrada até o momento em que a superfície do solo torna-se empoçada (Ip) é dada por:

$$
\mathrm{Ip}=\frac{\left(\Delta \theta_{1}\right) \varphi_{\mathrm{f} 1}}{\left(\frac{\mathrm{ip}}{\mathrm{Kt}_{1}}-1\right)} \quad \text { para } \mathrm{Ip} \leq \mathrm{I} 1
$$

$\mathrm{Ip}=\left[\frac{\Delta \theta_{2}\left(\mathrm{~L}_{1}+\varphi_{\mathrm{f} 2}\right)-\mathrm{L}_{1}\left(\Delta \theta_{2}\right)\left(\frac{\mathrm{ip}}{\mathrm{Kt}_{2}}\right)}{\left(\frac{\mathrm{ip}}{\mathrm{Kt}_{2}}\right)-1}\right]+\mathrm{I}_{1} \quad$ para Ip $>\mathrm{I}_{1}$

em que:

$\mathrm{I}_{1}=\mathrm{L}_{1}\left(\Delta \theta_{1}\right)$ - lâmina infiltrada na camada $1, \mathrm{~mm}$

$\Delta \theta_{1}$ e $\Delta \theta_{2}$ - déficit de umidade inicial nas camadas 1 e 2 , respectivamente, adimensional

$\varphi_{\mathrm{f} 1}$ e $\varphi_{\mathrm{f} 2}$ - potencial matricial do solo na frente de umedecimento nas camadas $1 \mathrm{e} 2$, respectivamente, $\mathrm{mm}$

$\mathrm{Kt}_{1}$ e $\mathrm{Kt}_{2}$ - condutividade hidráulica na zona de transmissão, camadas 1 e 2 , respectivamente, $\mathrm{mm} \mathrm{h}^{-1}$

$\mathrm{L}_{1}$ - espessura da camada $1, \mathrm{~mm}$

O tempo de empoçamento (tp), em horas, é dado por:

$$
\mathrm{tp}=\frac{\mathrm{Ip}}{\mathrm{ip}}
$$

A partir de $\mathrm{t}>\mathrm{tp}$, a taxa de infiltração (Ti) passa a ser igual à capacidade de infiltração (Ci) e dependente da lâmina infiltrada no tempo $(\mathrm{I}(\mathrm{t}))$, sendo dada por:

$$
\begin{aligned}
& \operatorname{Ti}(t)=\mathrm{Ci}(\mathrm{t})=\mathrm{Kt}_{1}\left(1+\frac{\left(\Delta \theta_{1}\right) \varphi_{\mathrm{f} 1}}{\mathrm{I}(\mathrm{t})}\right) \quad \operatorname{para} \mathrm{I}(\mathrm{t}) \leq \mathrm{I}_{1} \\
& \operatorname{Ti}(\mathrm{t})=\mathrm{Ci}(\mathrm{t})=\mathrm{Kt}_{2}\left(\frac{\mathrm{H}+\mathrm{I}(\mathrm{t})-\mathrm{I}_{1}}{\mathrm{E}+\mathrm{I}(\mathrm{t})-\mathrm{I}_{1}}\right) \quad \operatorname{para} \mathrm{I}(\mathrm{t})>\mathrm{I}_{1}
\end{aligned}
$$

em que:

$$
\mathrm{H}-\Delta \theta_{2}\left(\mathrm{~L}_{1}+\varphi_{\mathrm{f} 2}\right) \mathrm{e} \mathrm{E}=\mathrm{L}_{1}\left(\Delta \theta_{2}\right)\left(\mathrm{Kt}_{2} / \mathrm{Kt}_{1}\right)
$$

Para os perfis de precipitação com intensidade variada ao longo do tempo o evento de precipitação foi dividido em pequenos intervalos de tempo, sendo que para cada intervalo a intensidade de precipitação foi considerada constante e se calculou, em cada intervalo, a capacidade de infiltração de água no solo ( $\mathrm{Ci}$ ) com uso das equações de GAML e GAML modificada por Moore (1981), e comparada com a intensidade de precipitação (ip). Na sequência é descrita a rotina de cálculos utilizada:

Condições iniciais:

No tempo $\mathrm{t}_{\mathrm{n}}$, para $\mathrm{n}=0$; sendo $\mathrm{n}$ o índice indicador do tempo, tem-se:

$$
\mathrm{I}\left(\mathrm{t}_{\mathrm{o}}\right)=0 ; \mathrm{CI}\left(\mathrm{t}_{\mathrm{o}}\right) \sim \infty ; \operatorname{Ti}\left(\mathrm{t}_{\mathrm{o}}\right)=\mathrm{ip}
$$


Para $n>0$ tem-se:

$$
I\left(t_{n}\right)=\left[\operatorname{ip}_{n}\left(t_{n}-t_{n-1}\right)\right]+I\left(t_{n-1}\right)
$$

A capacidade de infiltração é dada pelas Eq. 5 ou 6.

Enquanto $\mathrm{Ci}>\mathrm{ip} ; \mathrm{Ti}$ = ip e seguiu-se o mesmo procedimento para o próximo intervalo de tempo. No primeiro momento em que $\mathrm{Ci}<\mathrm{ip}$, ocorre o empoçamento da água na superfície do solo e se considera que $\mathrm{Ti}=\mathrm{Ci}$. O tempo de empoçamento $(\mathrm{tp})$ foi determinado como ocorrendo neste momento, ou seja, tn = tp e a lâmina infiltrada até o início do empoçamento (Ip) é igual a I(tn).

A partir deste momento seguiu-se o mesmo procedimento adotado para a simulação com uso do perfil de precipitação constante (Eqs. 1 a 6), sendo que a cada intervalo de tempo foi realizada a comparação de Ci com ip e verificada a condição de ocorrência ou não do empoçamento na superfície do solo.

Para determinar o valor da condutividade hidráulica da crosta (Kc), ou seja, da camada 1, empregada a equação de Brakensiek \& Rawls (1983), que possibilita determinar a condutividade hidráulica equivalente $(\mathrm{Ke})$ :

$$
\mathrm{Ke}=\frac{\mathrm{L}}{\frac{\mathrm{L}-\mathrm{Zc}}{\mathrm{Ksc}}+\frac{\mathrm{Zc}}{\mathrm{Kc}}}
$$

em que:

Ke - condutividade hidráulica equivalente na zona de transmissão, $\mathrm{mm} \mathrm{h}^{-1}$

L - espessura da zona de transmissão, mm

Zc - espessura da crosta, mm

$\mathrm{Kc}$ - condutividade hidráulica da crosta, $\mathrm{mm} \mathrm{h}^{-1}$

Ksc- condutividade hidráulica da camada abaixo da crosta (subcrosta), $\mathrm{mm} \mathrm{h}^{-1}$

A espessura da crosta foi admitida como tendo um valor de $5 \mathrm{~mm}$, conforme recomendado por Brakensiek \& Rawls (1983) para a simulação do processo de infiltração em solos encrostados. Zonta et al. (2009) concluíram, em trabalho de simulação do processo de infiltração de água em solos encrostados, que a variação na espessura da crosta de 1 a 10 $\mathrm{mm}$ não influenciou significativamente nos resultados da simulação da taxa de infiltração com uso do modelo de GAML modificado por Moore (1981).

$\mathrm{O}$ valor da condutividade hidráulica equivalente $(\mathrm{Ke})$ foi admitido como sendo a taxa de infiltração estável média obtida em ensaios de infiltração em solo sem cobertura, igual a 15 $\mathrm{mm} \mathrm{h}^{-1}$. Com uso dos valores do conteúdo de água no solo determinados pela sondas de TDR a cada minuto durante os ensaios de infiltração, constatou-se que na maioria dos ensaios de infiltração em solo nu a frente de umedecimento alcançou apenas os $10 \mathrm{~cm}$ de profundidade sendo este valor considerado, então, como a espessura total da camada de solo (L), que é igual à espessura da zona de transmissão.

A condutividade hidráulica da subcrosta (Ksc) foi determinada de duas maneiras: para isto foram realizados ensaios de infiltração em solo com cobertura, ou seja, teoricamente sem o efeito do encrostamento superficial. $\mathrm{Na}$ primeira forma, Ksc foi igual à Tie obtida em ensaios de infiltração em solo com cobertura, $60 \mathrm{~mm} \mathrm{~h}^{-1}$ e na segunda Ksc foi igual à condutividade hidráulica do solo saturado $\left(\mathrm{K}_{0}\right)$ do horizonte $\mathrm{A}, 40 \mathrm{~mm} \mathrm{~h}^{-1}$.

Uma vez conhecida a espessura da crosta $(\mathrm{Zc})$ e da subcrosta $(Z s c=L-Z c)$, a condutividade hidráulica da subcrosta (Ksc) e a condutividade hidráulica equivalente (Ke), a condutividade hidráulica da crosta $(\mathrm{Kc})$ pode ser determinada por meio da Eq. 8 (Tabela 2).

Tabela 2. Valores da resistência hidráulica da crosta $(\mathrm{Rh}=\mathrm{Zc} / \mathrm{Kc})$, da condutividade hidráulica da crosta saturada (Kc), da espessura da crosta (Zc) e da subcrosta (Zsc), da condutividade hidráulica da subcrosta saturada (Ksc) e da condutividade hidráulica saturada equivalente

\begin{tabular}{|c|c|c|c|c|c|c|c|}
\hline \multirow{2}{*}{ Hipótese } & \multicolumn{2}{|c|}{$\begin{array}{l}\text { Zona de } \\
\text { transmissão }\end{array}$} & \multicolumn{3}{|c|}{ Crosta } & \multicolumn{2}{|c|}{ Sub-crosta } \\
\hline & $\begin{array}{c}\mathrm{Z} \\
\mathrm{mm}\end{array}$ & $\begin{array}{c}\mathrm{Ke} \\
\mathrm{mm} \mathrm{h}^{-1}\end{array}$ & $\begin{array}{c}\text { Rh } \\
\text { h }\end{array}$ & $\begin{array}{c}\mathrm{Kc} \\
\mathrm{mm} \mathrm{h} \mathbf{h}^{-1}\end{array}$ & $\begin{array}{l}\mathrm{Zc} \\
\mathrm{mm}\end{array}$ & $\begin{array}{c}\mathrm{Ksc} \\
\mathrm{mm} \mathrm{h}^{-1}\end{array}$ & $\begin{array}{l}\text { Zsc } \\
\mathrm{mm}\end{array}$ \\
\hline $\mathrm{Ksc}=\mathrm{Tie}$ & 100 & 15,0 & 5,1 & 1,0 & 5,0 & 60,0 & 95 \\
\hline $\mathrm{KsC}=\mathrm{Ko}$ & 100 & 15,0 & 4,3 & 1,2 & 5,0 & 40,0 & 95 \\
\hline Média & & & 4,7 & 1,1 & & & \\
\hline
\end{tabular}
$(\mathrm{Ke})$

Na simulação do processo de infiltração os parâmetros de entrada do modelo de GAML (condutividade hidráulica na zona de transmissão $(\mathrm{Kt})$, potencial mátrico na frente de umedecimento $\left(\varphi_{\mathrm{f}}\right)$ e umidade de saturação na zona de transmissão $\left.(\theta t)\right)$ foram determinados da seguinte forma:

O parâmetro $\theta t$ foi considerado igual ao conteúdo de água no solo obtido em campo $(\theta \mathrm{w})$ ao final dos ensaios de infiltração, conforme proposto por Slack (1980), sendo determinados com o uso das sondas de TDR instaladas no perfil do solo, apresentando valor médio de $0,418 \mathrm{~cm}^{3} \mathrm{~cm}^{-3}$, ou seja, $0,91 \theta \mathrm{s}$.

$\mathrm{O}$ valor de $\varphi_{\mathrm{f}}$ foi determinado com uso da equação proposta por Cecílio et al. (2007) pois, segundo os próprios autores e Zonta et al. (2010), esta é a proposta que apresentou os melhores resultados em seus estudos. $\mathrm{O}$ valor de $\varphi_{\mathrm{f}}$ é dado pela Eq. 9 .

$$
\varphi_{\mathrm{t}}=\frac{\varphi_{\mathrm{b}}(2+3 \lambda)\left[\mathrm{K}_{\mathrm{r}}\left(\theta_{\mathrm{w}}\right)^{\frac{3 \lambda+1}{3 \lambda+2}}-\mathrm{K}_{\mathrm{r}}\left(\theta_{\mathrm{i}}\right)^{\left.\frac{3 \lambda+1}{3 \lambda+2}\right]}\right.}{\left[\mathrm{K}_{\mathrm{r}}\left(\theta_{\mathrm{w}}\right)-\mathrm{K}_{\mathrm{r}}\left(\theta_{\mathrm{i}}\right)\right][3 \lambda+1]}
$$

em que:

$$
\mathrm{K}_{\mathrm{r}}(\theta)=\mathrm{K}_{0}\left(\frac{\varphi_{\mathrm{b}}}{\varphi(\theta)}\right)^{2+3 \lambda}
$$

em que:

$\varphi_{\mathrm{b}}$ - potencial matricial de entrada de ar, mm

$\lambda$ - índice de distribuição de tamanho de poros, adimensional $\varphi(\theta)$ - potencial matricial referente ao conteúdo de água $(\theta)$

A condutividade hidráulica na zona de transmissão $(\mathrm{Kt})$ foi considerada com diferentes valores. Para o modelo de 
GAML o valor de Kt foi considerado igual à Tie obtida em solo com e sem cobertura, 60 e $15 \mathrm{~mm} \mathrm{~h}^{-1}$, respectivamente, conforme proposta de Silva \& Kato (1998) e Kt igual à condutividade hidráulica do solo saturado $\left(\mathrm{K}_{0}\right)$ para o horizonte A, $40 \mathrm{~mm} \mathrm{~h}^{-1}$.

Para o modelo de GAML modificado por Moore (1981), na camada de encrostamento o valor de Kt foi considerado como sendo o valor médio da condutividade hidráulica da crosta (Kc), determinada pelas duas metodologias aqui propostas, igual a $1,1 \mathrm{~mm} \mathrm{~h}^{-1}$ (Tabela 2), igual a 0,1 e $0,25 \times \mathrm{K}_{0}$ do horizonte A, sendo tais valores determinados em simulações prévias. Para o valor de Kt da subcrosta, foi utilizado o valor de $0,5 \times \mathrm{K}_{0}$ do horizonte A do solo, conforme recomendação de Rawls et al. (1983).

Na Tabela 3 são apresentadas as combinações de propostas para determinação dos parâmetros de entrada do modelo de GAML.

Tabela 3. Combinações de proposições utilizadas nas simulações realizadas com o modelo de GAML

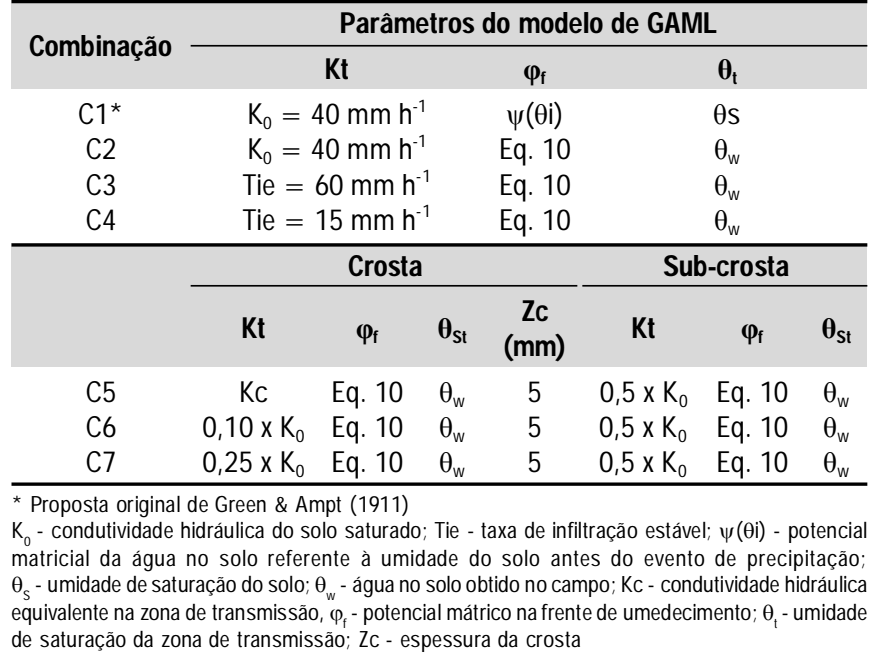

Realizou-se a avaliação do desempenho do modelo de GAML comparando-se as curvas experimentais e simuladas da taxa de infiltração e infiltração acumulada através do índice de confiança (c) obtido pelo produto entre o coeficiente de correlação de Pearson (r) e o índice de concordância (d), proposto por Willmott (1981), obtido pela Eq. 11.

$$
\mathrm{d}=1-\frac{\sum_{\mathrm{i}=1}^{\mathrm{J}}\left(\left|\mathrm{O}_{\mathrm{i}}-\mathrm{E}_{\mathrm{i}}\right|\right)^{2}}{\sum_{\mathrm{i}=1}^{\mathrm{J}}\left(\left|\mathrm{E}_{\mathrm{i}}=\overline{\mathrm{O}}\right|+\left|\mathrm{O}_{\mathrm{i}}-\overline{\mathrm{O}}\right|\right)^{2}}
$$

em que:

$$
\begin{array}{ll}
\text { J } & \text { - número de observações } \\
\text { Oi } & \text { - valor observado experimentalmente } \\
\underline{\text { E }} & \text { - valor estimado pelo modelo } \\
\mathrm{O} & \text { - média dos valores observados experimentalmente }
\end{array}
$$

O desempenho do modelo avaliado pelo uso do índice de confiança (c), foi classificado de acordo com a proposta de Camargo \& Sentelhas (1997), conforme apresentado na Tabela 4.
Tabela 4. Análise do desempenho do modelo com base no índice de confiança ajustado

\begin{tabular}{cl}
\hline Valor de c & Desempenho \\
$>0,85$ & Ótimo \\
0,76 a 0,85 & Muito bom \\
0,66 a 0,75 & Bom \\
0,61 a 0,65 & Mediano \\
0,51 a 0,60 & Sofrível \\
0,41 a 0,50 & Mau \\
$\leq 0,40$ & Péssimo \\
\hline
\end{tabular}

\section{RESULTADOS E DISCUSSÃO}

Na Tabela 5 é apresentado o desempenho das simulações realizadas na estimativa de Ti e I feitas nas três aplicações sucessivas com os quatro perfis de precipitação utilizados. Nas

\begin{tabular}{|c|c|c|c|c|c|}
\hline \multirow{2}{*}{\multicolumn{2}{|c|}{ Combinação Parâmetro }} & \multicolumn{4}{|c|}{ Perfil de precipitação } \\
\hline & & Constante & $\begin{array}{l}\text { Exponencial } \\
\text { decrescente }\end{array}$ & \multicolumn{2}{|c|}{ Duplo exponencial } \\
\hline \multirow[b]{3}{*}{$\mathrm{C} 1$} & & \multicolumn{4}{|c|}{$1 \stackrel{\text { a }}{\text { Aplicação }}$} \\
\hline & $\mathrm{Ti}$ & Péssimo & Ótimo & Ótimo & Sofrivel \\
\hline & I & Ótimo & Ótimo & Ótimo & Ótimo \\
\hline \multirow{2}{*}{$\mathrm{C} 2$} & $\mathrm{Ti}$ & Mediano & Bom & Péssimo & Péssimo \\
\hline & I & Ótimo & Ótimo & Ótimo & Ótimo \\
\hline \multirow{2}{*}{$\mathrm{C} 3$} & $\mathrm{Ti}$ & Muito Bom & Ótimo & Muito Bom & Mediano \\
\hline & I & Ótimo & Ótimo & Ótimo & Ótimo \\
\hline \multirow{2}{*}{ C4 } & $\mathrm{Ti}$ & Péssimo & Mau & Péssimo & Péssimo \\
\hline & I & Bom & Mediano & Bom & Bom \\
\hline \multirow[b]{3}{*}{$\mathrm{C} 1$} & & \multicolumn{4}{|c|}{$2^{\text {a }}$ Aplicação } \\
\hline & $\mathrm{Ti}$ & Péssimo & Sofrível & Péssimo & Péssimo \\
\hline & 1 & Mediano & Péssimo & Sofrível & Péssimo \\
\hline \multirow{2}{*}{$\mathrm{C} 2$} & $\mathrm{Ti}$ & Bom & Bom & Mau & Péssimo \\
\hline & I & Ótimo & Mau & Muito bom & Sofrível \\
\hline \multirow{2}{*}{ C3 } & $\mathrm{Ti}$ & Mediano & Sofrível & Péssimo & Péssimo \\
\hline & 1 & Bom & Péssimo & Bom & Mau \\
\hline \multirow{2}{*}{ C4 } & $\mathrm{Ti}$ & Muito bom & Bom & Bom & Ótimo \\
\hline & I & Muito bom & Muito bom & Ótimo & Ótimo \\
\hline \multirow{2}{*}{ C5 } & $\mathrm{Ti}$ & Péssimo & Sofrível & Péssimo & Péssimo \\
\hline & I & Mediano & Ótimo & Bom & Ótimo \\
\hline \multirow{2}{*}{$\mathrm{C} 6$} & $\mathrm{Ti}$ & Bom & Bom & Bom & Ótimo \\
\hline & I & Muito bom & Ótimo & Ótimo & Ótimo \\
\hline \multirow{3}{*}{$\mathrm{C7}$} & $\mathrm{Ti}$ & Muito bom & Muito bom & Muito bom & Muito bom \\
\hline & I & Ótimo & Muito bom & Ótimo & Muito bom \\
\hline & & & $3 \mathrm{a} \mathrm{Apl}$ & licação & \\
\hline \multirow{2}{*}{$\mathrm{C} 1$} & $\mathrm{Ti}$ & Péssimo & Péssimo & Péssimo & Péssimo \\
\hline & I & Sofrível & Bom & Mau & Sofrível \\
\hline \multirow{2}{*}{ C2 } & $\mathrm{Ti}$ & Muito bom & Mau & Sofrível & Péssimo \\
\hline & I & Muito bom & Muito bom & Bom & Bom \\
\hline \multirow{2}{*}{ C3 } & $\mathrm{Ti}$ & Mau & Péssimo & Péssimo & Péssimo \\
\hline & 1 & Mediano & Bom & Sofrível & Sofrível \\
\hline \multirow{2}{*}{ C4 } & $\mathrm{Ti}$ & Muito bom & Bom & Ótimo & Bom \\
\hline & 1 & Ótimo & Muito bom & Ótimo & Ótimo \\
\hline \multirow{2}{*}{ C5 } & $\mathrm{Ti}$ & Péssimo & Sofrível & Péssimo & Péssimo \\
\hline & 1 & Mediano & Muito bom & Bom & Muito bom \\
\hline \multirow{2}{*}{$\mathrm{C} 6$} & $\mathrm{Ti}$ & Bom & Bom & Muito bom & Bom \\
\hline & 1 & Ótimo & Ótimo & Ótimo & Ótimo \\
\hline \multirow{2}{*}{$\mathrm{C} 7$} & $\mathrm{Ti}$ & Muito bom & Muito bom & Ótimo & Bom \\
\hline & I & Ótimo & Ótimo & Ótimo & Ótimo \\
\hline
\end{tabular}

Tabela 5. Desempenho do modelo de GAML na simulação da taxa de infiltração (Ti) e lâmina infiltrada (I), com base no índice de concordância (c), para ensaios de infiltração realizados com diferentes perfis de precipitação 
Figuras 3 a 6 se encontram os gráficos com as curvas da taxa de infiltração (Ti) e infiltração acumulada (I) no tempo, medidas e simuladas com as diferentes combinações de parâmetros de entrada do modelo de GAML, para as primeira e terceira aplicações, para os quatro perfis de precipitação utilizados.

De forma geral, para a $1^{\mathrm{a}}$ aplicação (Tabela 5), todas as combinações avaliadas apresentaram bons resultados na simulação de I, com desempenho acima de bom em pelo menos $75 \%$ dos casos. Para a simulação de Ti, os melhores resultados foram com a combinação C3, que apresentou desempenho muito bom ou superior a $75 \%$ dos casos. As demais combinações

A.

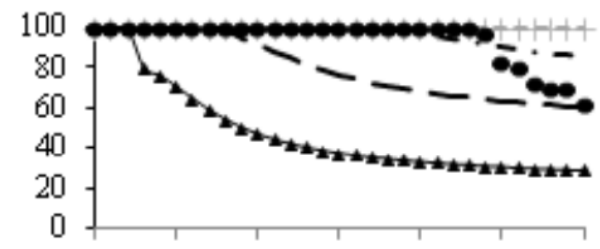

B.

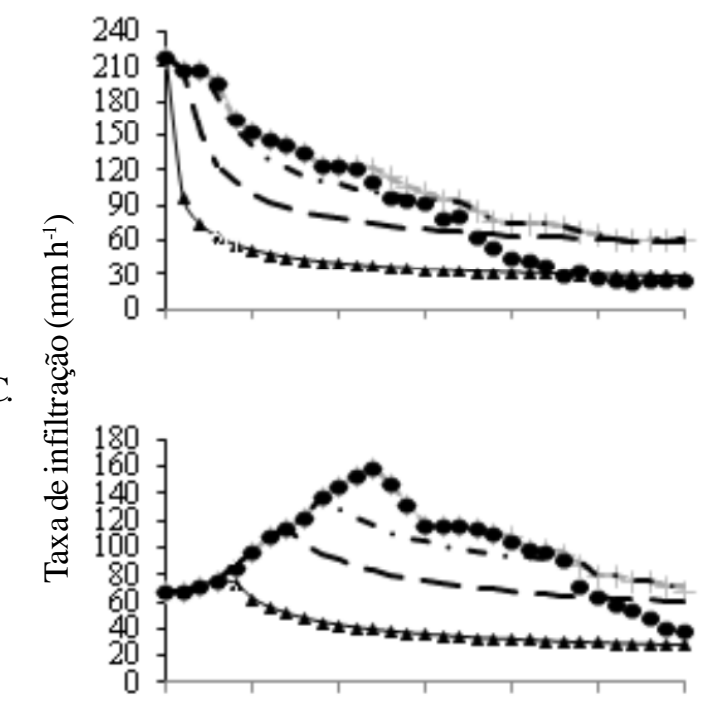

D.

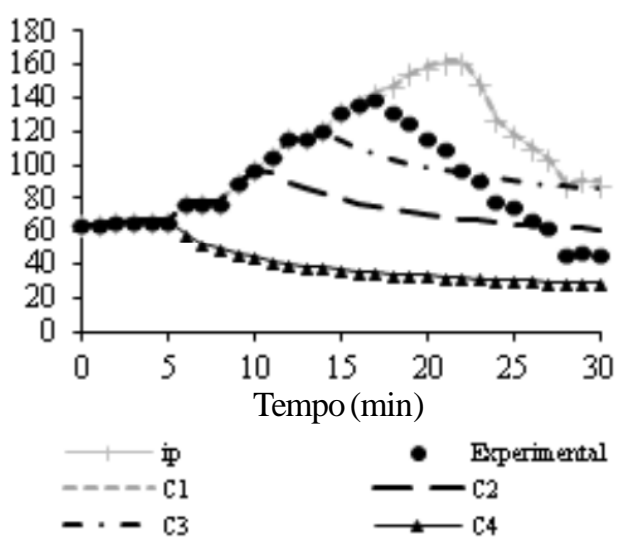

Fig a 3. Taxa de infiltração medida e estimada pelo modelo de G AM L usando-se diferentes combinações de seus parâmetros de entrada, 1a aplicação, perfis de precipitação constante $(A)$, exponencial decrescente $(B)$, duplo exponencial adiantado (C) e atrasado (D)
A.

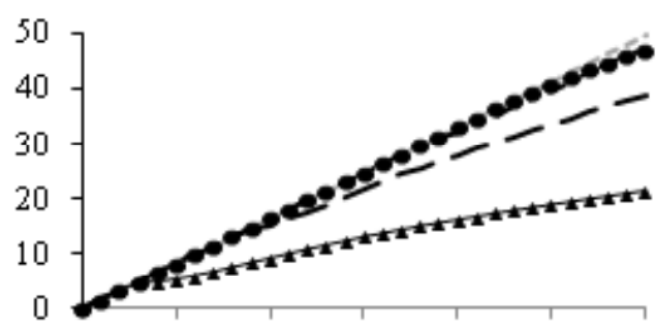

B.

C.

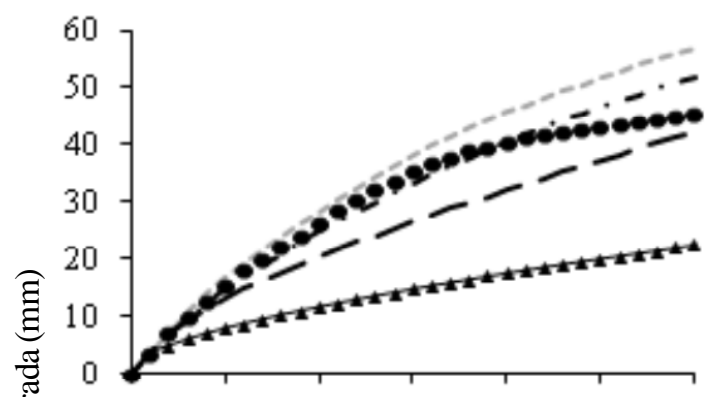

C. .

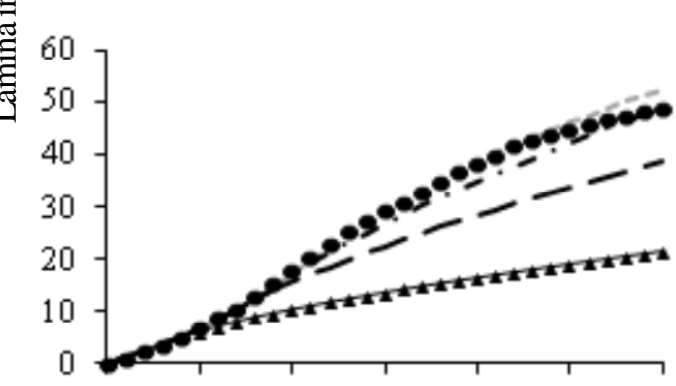

D.

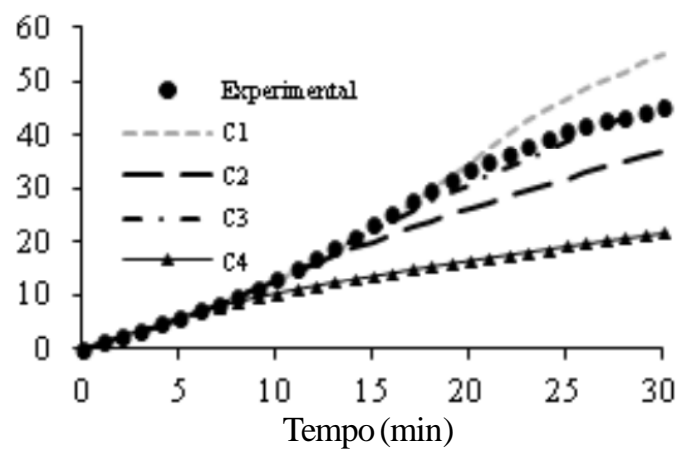

Figura 4. Lâmina infiltrada medida e estimada pelo modelo de GAM L usando-se diferentes combinações de seus parâmetros de entrada, 1a aplicação, perfis de precipitação constante $(A)$, exponencial decrescente $(B)$, duplo exponencial adiantado (C) e atrasado (D)

(C1, C2 e C4) não apresentaram bons resultados, com desempenho abaixo de sofrível em pelo menos $50 \%$ dos casos para cada combinação. Os resultados corroboram com os obtidos por Mello (2003), Cecílio et al. (2007) e Zonta et al. (2010), que encontraram melhor desempenho do modelo de GAML na simulação da lâmina infiltrada, se comparado com a taxa de infiltração.

O bom desempenho do modelo de GAML na simulação da lâmina infiltrada com uso das combinações $\mathrm{C} 1$ a $\mathrm{C} 4$ pode ser 
A.

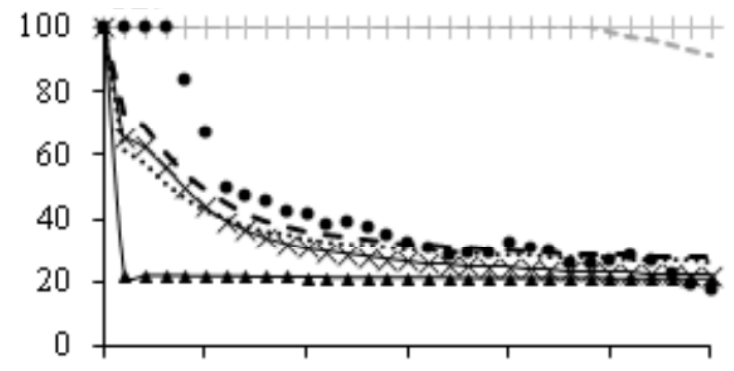

B.

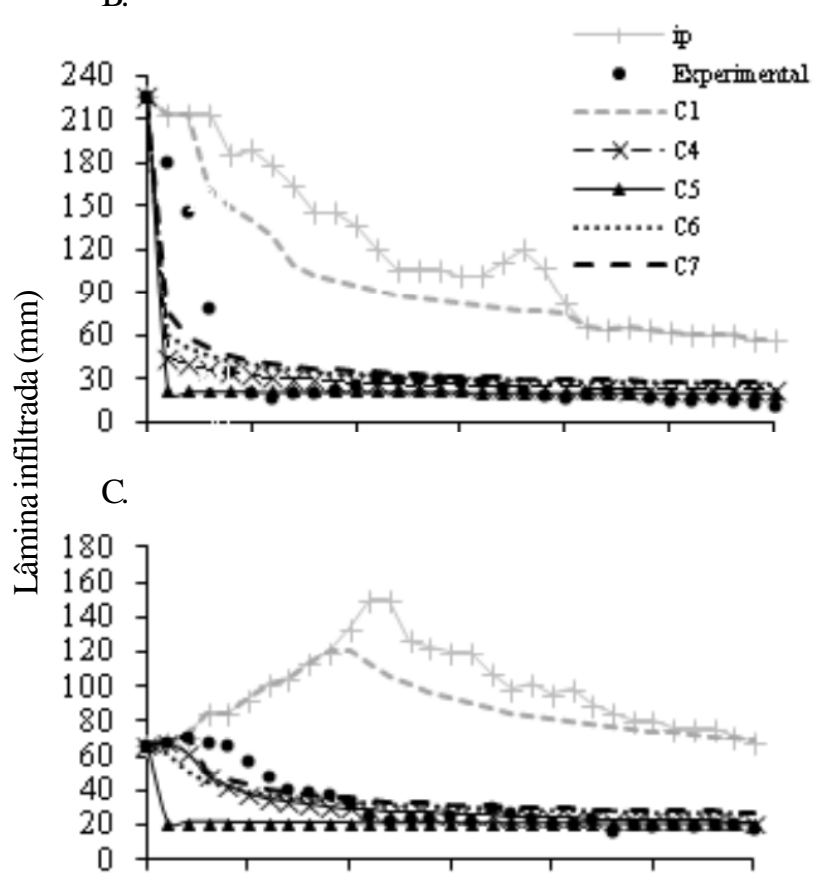

D.

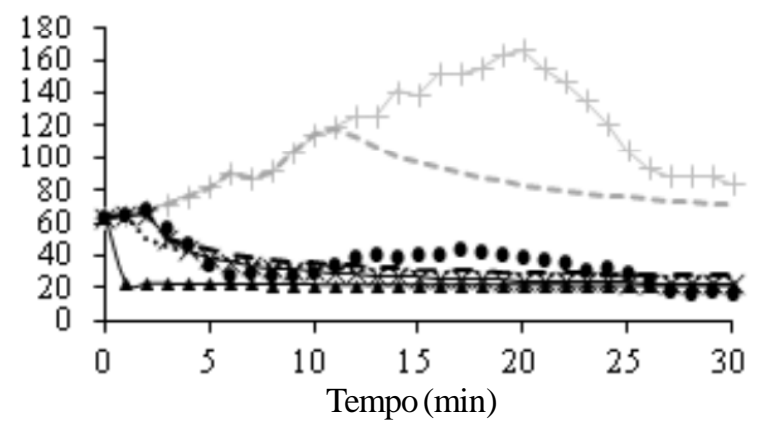

Figura 5. Taxa de infiltração medida e estimada pelo modelo de GAM L usando-se diferentes combinações de seus parâmetros de entrada, 3a aplicação, perfis de precipitação constante (A), exponencial decrescente (B), duplo exponencial adiantado (C) e atrasado (D)

atribuído principalmente à elevada capacidade de infiltração apresentada por este solo, visto que na $1^{a}$ aplicação quase não houve formação de escoamento superficial, isto é, quase toda precipitação aplicada se infiltrou no perfil do solo. Wells et al. (1986) afirmam, testando diversos modelos de simulação do processo de infiltração de água no solo, dentre eles o de GAML, que geralmente o modelo tende a superestimar os valores da $\mathrm{Ti}$
A.

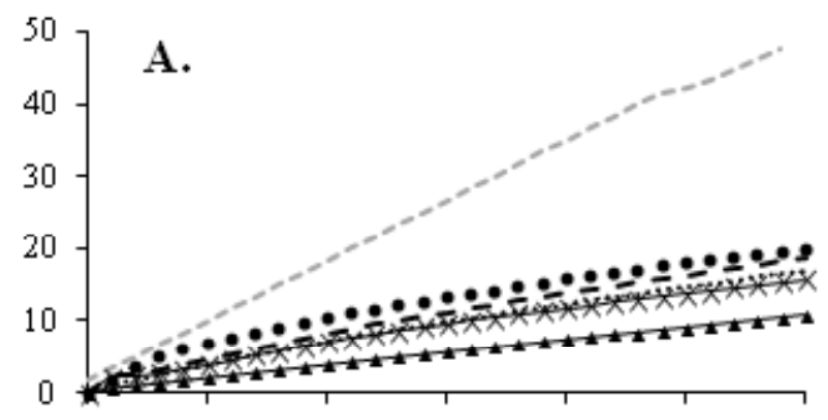

B.

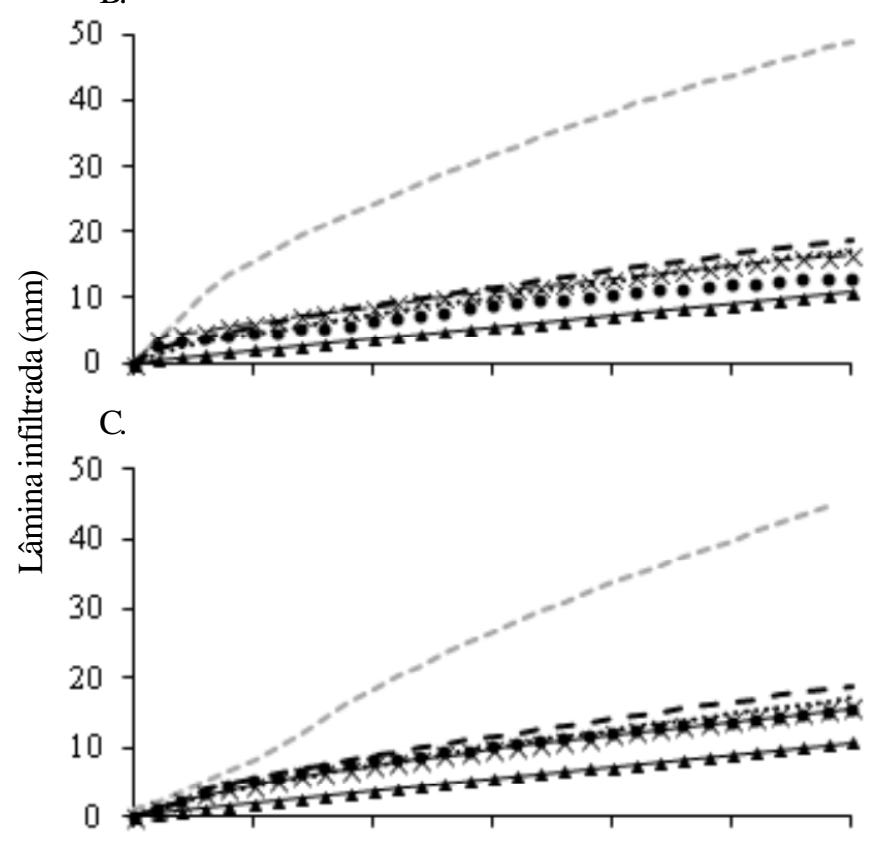

D.

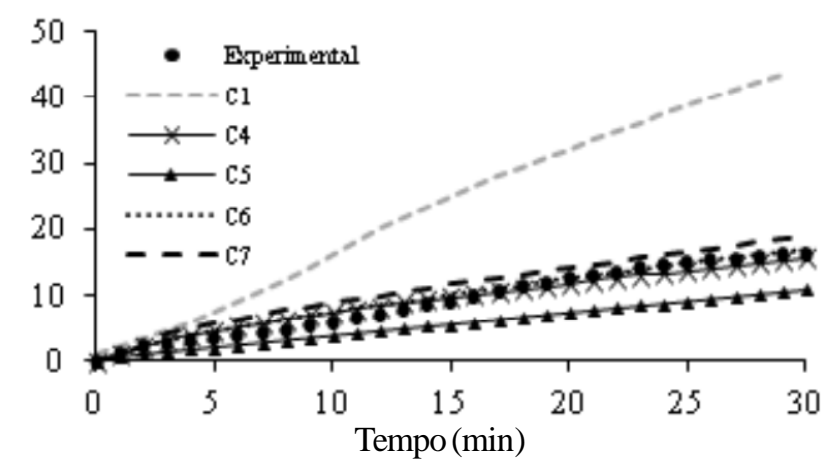

Figura 6. Lâmina infiltrada medida e estimada pelo modelo de G AML usando-se diferentes combinações de seus parâmetros de entrada, 3a aplicação, perfis de precipitação constante $(A)$, exponencial decrescente $(B)$, duplo exponencial adiantado (C) e atrasado (D)

ao longo do tempo; assim, em casos de elevada capacidade de infiltração de água no solo, o modelo tende a apresentar bons resultados.

Dentre tais combinações, os melhores resultados foram obtidos com o uso da Tie (obtida em solo com cobertura, ou seja, sem encrostamento superficial) como valor de Kt, e o valor de $\varphi_{\mathrm{f}}$ determinado com uso da equação de Cecílio et al. (2007), 
ou seja, C3. Esta combinação proporcionou a melhor simulação do tempo de empoçamento, com pequena superestimativa nos valores de Ti no final dos ensaios. Esta foi a combinação que melhor simulou os valores de I, com as curvas de I medidas e simuladas se sobrepondo, praticamente, ao longo do tempo. Desta forma, esta solução pode ser considerada um simplificador muito grande da equação de GAML para solos estratificados em razão da não necessidade da determinação da condutividade hidráulica do solo saturado para cada estrato.

Esses resultados demonstram que o uso da Tie como parâmetro de entrada do modelo de GAML se apresenta como boa alternativa devido, sobremaneira, à maior confiabilidade na determinação de seus valores em relação à determinação de $\mathrm{K}_{0}$ (Cecílio et al., 2003). Silva \& Kato (1998) e Zonta et al. (2010) também encontraram bom resultados na simulação do processo de infiltração de água no solo com uso da Tie como parâmetro de entrada do modelo de GAML.

Fica a ressalva de que os ensaios de infiltração realizados na $1^{\text {a }}$ aplicação apresentaram curta duração (30 min), não apresentando, portanto, tempo suficiente para que fosse alcançado o valor da Tie. Desta forma não é possível extrapolar a afirmação acima para ensaios de longa duração, nos quais ocorre um decaimento mais acentuado nos valores da Ti devido ao avanço da frente de umedecimento em maiores profundidades, tornando o gradiente hidráulico unitário e a formação de encrostamento superficial. Nessas condições, mesmo com a utilização das propostas de correção dos parâmetros de entrada do modelo este poderia não apresentar bons resultados, conforme afirmam Zonta et al. (2010).

Zonta et al. (2010), trabalhando no mesmo solo, encontraram resultados similares na simulação do processo de infiltração com uso do modelo de GAML em sua forma original, para eventos de precipitação de curta duração. Estes autores atribuíram o resultado ao baixo conteúdo inicial de água no solo e ao consequeente potencial matricial reduzido. Deve-se ressaltar que para ensaios com maior duração os mesmos autores não encontraram bons resultados na simulação do processo de infiltração com uso do modelo de GAML em sua forma original.

Como para a $2^{\mathrm{a}}$ e $3^{\mathrm{a}}$ aplicações os resultados foram semelhantes, foram apresentados somente os gráficos para a $3^{\mathrm{a}}$ aplicação (Figuras 5 e 6). Para esta aplicação, a combinação C1, que utiliza os parâmetros originais da equação de GAML superestimou os valores de Ti e I ao longo do tempo para todos os perfis de precipitação, assim não apresentou bom desempenho (Tabela 5). Zonta et al. (2010) concluíram que, na sua forma original, a equação de GAML não apresentou bons resultados na simulação do processo de infiltração, superestimando as taxas de infiltração e infiltração acumulada em ensaios de infiltração de longa duração, cuja Tie é alcançada, como no caso da $2^{\mathrm{a}}$ e $3^{\mathrm{a}}$ aplicações deste experimento.

Nas segunda e terceira aplicações o modelo de GAML apresentou, para um único estrato, resultados mas somente com uso da combinação $\mathrm{C} 4$, com desempenho bom ou superior em todos os casos para Ti e I. De forma geral, as demais combinações não apresentaram bons resultados, principalmente na simulação de Ti, com desempenho acima de bom em no máximo 37,5\% dos casos para a combinação C2.
Se comparadas as combinações C2, C3 e C4, cuja diferença nos parâmetros de entrada está no valor de Kt, observa-se que os melhores resultados obtidos pela combinação C4 são devidos ao uso do valor da Tie obtida em solo nu, como valor de Kt. Desta forma, fica a ressalva de que, apesar de indicado o uso da Tie em substituição a $\mathrm{K}_{0}$ como valor de $\mathrm{Kt}$, esta deve ser obtida nas mesmas condições encontradas em campo.

Este resultado sugere, para a simulação do processo de infiltração de água em solos com encrostamento superficial utilizando-se o modelo de GAML para um único estrato de solo, que o encrostamento superficial deveria ser considerado no modelo trabalhando-se com o valor de Kt igual ao da Tie, esta obtida em ensaios com simulador de chuvas em solo sem cobertura.

Os resultados obtidos com as combinações C4, C6 e C7 foram bem semelhantes apresentando subestimativas nos valores de Ti e I, os quais foram mais evidentes nos ensaios com perfil de precipitação constante em virtude, sobremaneira, da subestimativa nos valores do tempo de empoçamento de vez que, após queda acentuada nos valores de Ti medidos, os valores foram bem simulados. Para os perfis de precipitação com intensidade variada ao longo do tempo, o momento do empoçamento da superfície do solo foi estimado satisfatoriamente, em que as simulações apresentaram resultado melhor, se comparado com o do perfil de precipitação constante.

Comparando-se as combinações de parâmetros de entrada para o modelo de GAML modificado por Moore (1981) (C5, C6 e C7), nas quais foi incluída a camada de encrostamento superficial no modelo, a combinação C5 não apresentou bons resultados no geral, com desempenho abaixo de sofrível em $100 \%$ dos casos para Ti, simulando bem os valores de I, com desempenho acima de bom em $75 \%$ dos casos (Tabela 5). As combinações C6 e C7 apresentaram bons resultados, com desempenho acima de bom em $100 \%$ dos casos, tanto para Ti como para I.

A diferença entre tais combinações reside no valor de Kt da crosta. Para a combinação C5 foi utilizado o valor de $\mathrm{Kt}=\mathrm{Kc}$ determinado a partir de dados experimentais, iguais a $1,1 \mathrm{~mm} \mathrm{~h}^{-1}$. Brandão et al. (2006) encontraram valores de Kc iguais a 0,9 $\mathrm{mm} \mathrm{h}^{-1}$, para um argissolo com $500 \mathrm{~J} \mathrm{~m}^{-2}$ de energia cinética acumulada da chuva, sendo que o solo em estudo possui características texturais parecidas com as utilizadas pelos autores citados. Neste experimento a energia cinética acumulada da chuva ao final da terceira aplicação sucessiva da precipitação foi, em média, igual a $465 \mathrm{~J} \mathrm{~m}^{-2}$ já nas combinações C6 e C7 o valor de $\mathrm{Kc}$ foi igual a 0,1 e 0,25 x K, respectivamente, indicando um valor de Kc igual a 4 e $10 \mathrm{~mm} \mathrm{~h}^{-1}$ para C6 e C7, valores esses escolhidos com base em simulações prévias.

Nota-se, então, que a combinação C5 apresentou um decréscimo acentuado da Ti logo no início do processo de infiltração, subestimando os valores iniciais de Ti e I e, com o passar do tempo, os valores apresentaram bom ajuste (Figura 5). Já as combinações C6 e C7 apresentaram um ajuste melhor aos dados experimentais, desde o início dos ensaios de infiltração; daí, portanto, os melhores desempenhos dessas combinações. 
Para a simulação com o modelo de GAML modificado por Moore (1981) para solos estratificados, sugere-se que a camada superficial seja considerada como sendo a camada de encrostamento, com condutividade hidráulica da crosta (Kc) variando de 10 a $25 \%$ da condutividade hidráulica do solo saturado $\left(\mathrm{K}_{0}\right)$, e o valor de $\mathrm{Kt}$ da segunda camada igual a $0,5 \mathrm{x}$ $\mathrm{K}_{0}$, com espessura desta crosta, para as condições deste experimento, de $5 \mathrm{~mm}$.

Os resultados demonstram que, apesar de os valores de Kc obtidos em campo serem próximos aos encontrados por outros pesquisadores, como Brandão et al. (2006), seu uso no modelo de GAML não acarreta em bons resultados simulados de Ti. De acordo com os resultados, o valor de Kc para a simulação com uso do modelo de GAML modificado por Moore (1981), para as condições experimentais descritas, deve variar de 10 a $25 \%$ do valor de $\mathrm{K}_{0}$.

Observa-se que, de forma geral, os melhores resultados para as segunda e terceira aplicações foram obtidos com as combinações que consideram o efeito do encrostamento superficial, vindo de acordo com as afirmações de Moore (1981).

Na combinação C4 esta consideração é feita com o uso da Tie obtida em solo nu como parâmetro de entrada, enquanto nas combinações C6 e C7 foi utilizado o modelo para solos estratificados, considerando-se a camada de encrostamento como sendo a camada superficial. As combinações C1 a C3, que não consideram a formação de encrostamento superficial no modelo de GAML, não apresentaram bons resultados na simulação do processo de infiltração para as segunda e terceira aplicações.

\section{ConClusõEs}

1. Nas condições de encrostamento superficial com as precipitações consecutivas, os resultados foram melhores quando este efeito foi introduzido no modelo, não se encontrando bons resultados nas simulações sem a consideração da crosta.

2. De modo geral, o modelo de GAML com modificações em seus parâmetros de entrada foi adequado para a simulação do processo de infiltração de água no solo, independente do perfil de precipitação aplicado, do conteúdo de água inicial do solo e da formação de encrostamento superficial.

\section{AgradeCiMENTOS}

À Fundação de Amparo à Pesquisa do Estado de Minas Gerais (FAPEMIG) pelo apoio e financiamento do projeto de pesquisa.

\section{LITERATURA CITADA}

Brakensiek, D. L.; Rawls, W. J. Agricultural management effects on soil water processes Part II: Green and Ampt parameters for crusting soils. Transactions of the ASAE, v.26, p.1753$1757,1983$.
Brandão, V. S.; Silva, D. D.; Ruiz, H. A.; Pruski, F. F.; Schaefer, C. E. G. R.; Martinez, M. A.; Menezes, S. J. M. C. Resistência hidráulica da crosta formada em solos submetidos a chuvas simuladas. Revista Brasileira de Ciência do Solo, v.30, p.1321, 2006.

Camargo, Â. P.; Sentelhas, P. C. Avaliação do desempenho de diferentes métodos de estimativa da evapotranspiração potencial no Estado de São Paulo, Brasil. Revista Brasileira de Agrometeorologia, v.5, p.89-97, 1997.

Cecílio, R. A.; Martinez, M. A.; Pruski, F. F.; Silva, D. D.; Ataíde, W .F. Substituição dos parâmetros do modelo de GreenAmpt-Mein-Larson para estimativa da infiltração em alguns solos do Brasil. Revista Brasileira de Ciência do Solo, v.31, p.1141-1151, 2007.

Cecílio, R. A.; Silva, D. D.; Pruski, F. F.; Martinez, M. A. Modelagem da infiltração de água no solo sob condições de estratificação utilizando-se a equação de Green-Ampt. Revista Brasileira de Engenharia Agrícola e Ambiental, v.7, p.415-422, 2003.

Chowdary, V. M.; Rao, M. D.; Jaiswal, C. S. Study of infiltration process under different experimental conditions. Agricultural Water Management, v.83, p.69-78, 2006.

Green, W. H.; Ampt, G. A. Studies on soil physics-1. The flow of air and water through soils. Journal of Agricultural Science, v.4, p.1-24, 1911.

Mein, R. G.; Larson, C. L. Modeling infiltration during a steady rain. Water Resources Research, v.9, p.384-394, 1973.

Mello, L. T. A. Avaliação de metodologias para obtenção dos parâmetros do modelo de Green-Ampt modificado por Mein e Larsson. Viçosa: UFV, 2003. 77p. Dissertação Mestrado

Meng, H.; Salas, J. D.; Green T. R.; Ahuja, LR. Scaling analysis of space-time infiltration based on the universal multifractal model. Journal of Hydrology, v.322, p.220-235, 2006.

Moore, I. D. Infiltration equations modified for surface effects. Journal of the Irrigation and Drainage Division, v.107, p.71$86,1981$.

Nahar, N.; Govindaraju, R. S.; Corradini, C.; Morbidelli, R. Role of run-on for describing field-scale infiltration and overland flow over spatially variable soils. Journal of Hydrology, v.286, p.36-51, 2004.

Rawls, W. J.; Brakensiek, D. L. A procedure to predict Green and Ampt infiltration parameters. In: ASAE Conference on Advances in Infiltration, 1983. Chicago. Proceedings... Chicago: ASAE, 1983. p.102-112.

Robinson, D. A.; Woodun, J. K. An experimental study of crust development on chalk downland soils and their impact on runoff and erosion. European Journal of Soil Science, v.59, p.784-798, 2008.

Schaefer, C. E. R.; Silva, D. D.; Paiva, K. W. N.; Pruski, F. F.; Albuquerque Filho, M. R.; Albuquerque, M. A. Perdas de solo, nutrientes, matéria orgânica e efeitos micro-estruturais em Argissolo Vermelho-Amarelo sob chuva simulada. Pesquisa Agropecuária Brasileira, v.37, p.669-678, 2002.

Shukla, M. K.; Lal, R.; Unkefer, P. Experimental evaluation of infiltration models for different land use and soil management systems. Soil Science, v.168, p.178-191, 2003. 
Silva, C. L.; Kato, E. Efeito do selamento superficial na condutividade hidráulica saturada da superfície de um solo sob cerrado. Pesquisa Agropecuária Brasileira, v.32, p.213220, 1997.

Silva, C. L.; Kato, E. Avaliação de modelos para a previsão da infiltração de água em solos sob cerrado. Pesquisa Agropecuária Brasileira, v.33, p.1149-1158, 1998.

Slack, D. C. Modeling infiltration under moving sprinkler irrigation systems. Transactions of the ASAE, v.23, p.596-600, 1980.

Souza, E. S.; Antonino, A. C. D.; Lima, J. R. S.; Gouveia Neto, G. C.; Silva, J. M.; Silva, I. F. Efeito do encrostamento superficial nas propriedades hidráulicas de um solo cultivado. Revista Brasileira de Ciências Agrárias, v.2, p.69-74, 2007.

Valentin, C.; Bresson, L. M. Morphology, genesis and classification of surface crusts in loamy and sandy soils. Geoderma, v.55, p.225-245, 1992.
Wells, L. G ; Ward, A. D.; Moore, I. D.; Phillips, R. E. Comparison of four models in characterizing through surface mine profiles. Transactions of the ASAE, v.29, p.785-793, 1986.

Willmott, C. J. On the validation of models. Physical Geography, v.2, p.184-194, 1981.

Zonta, J. H.; Martinez, M. A.; Pruski, F. F.; Silva, D. D.; Montebeller, C. A. Adequação dos parâmetros do modelo de Green-Ampt-Mein-Larson em condições de campo. Revista Brasileira de Engenharia Agrícola e Ambiental, v.14, p.1021-1029, 2010.

Zonta, J. H.; Martinez, M. A.; Viana, M. L.; Santos, M. R. Influência da espessura da crosta superficial na simulação do processo de infiltração de água no solo usando o modelo GAML modificado por Moore (1981). In: Simpósio da PósGraduação da UFV, 9, 2009, Viçosa. Anais... Viçosa: UFV, 2009. CD-Rom 